\title{
Postural Adjustments in Preterm Infants at 4 and 6 Months Post-Term During Voluntary Reaching in Supine Position
}

\author{
BJØRG FALLANG, OLA DIDRIK SAUGSTAD, AND MIJNA HADDERS-ALGRA \\ Oslo University College, Health Sciences, Physiotherapy Programme, Pilestredet 52, 0167 Oslo, Norway \\ [B.F.]; Department of Pediatric Research, The National Hospital, University of Oslo, 0027 Oslo, Norway \\ [O.D.S.]; University Hospital Groningen, Developmental Neurology, Hanzeplein 1, 9713 GZ Groningen,
}

The Netherlands [M.H.-A.]

\begin{abstract}
ABSTR
Gradually it is getting clear that motor development - in
particular balance control - in so-called "low-risk" preterm
infants often differs from that in full-term infants. However, little
is known on the etiology and pathophysiology of these problems.
The aim of this study was to evaluate postural behavior during
reaching by means of kinetic and kinematic measurements.
Preterm infants ( $n=32$ ) without cerebral palsy were investi-
gated longitudinally at the corrected ages of 4 and 6 mo. Thirteen
age-matched full-term infants served as controls. Cognitive and
motor development were assessed by means of the quality of
General Movements (GMs) at 4 mo and Bayley scales at 6 and
12 mo. The infants were lying supine on a forceplate reaching for
a toy and the kinetics of the total body's Center of Pressure
(COP) was measured in cranial-caudal and medial-lateral direc-
tion. The analysis focused on COP displacement, $V_{\text {max and }}$
oscillatory changes of the COP displacement during reaching.
The kinematic analysis of reaching focused on movement units,
$V_{\text {max }}$ and a compound kinematic variable reflecting the quality of
reaching. The results showed that preterm infants showed a
remarkable "still" postural behavior, which differed significantly
\end{abstract}
from the mobile COP behavior of the full-term infants. More "still" postural behavior at 6 mo was associated with a better quality of reaching movements and with normal GMs at 4 mo. We concluded that "still" postural behavior is an adequate postural strategy of preterm infants. But it might be that this postural behavior is an indicator of later dysfunction. (Pediatr Res 54: 826-833, 2003)

$\quad$ Abbreviations
COP, center of pressure
c-c, cranial-caudal direction
HR, high-risk
LR, low-risk
MDI, Mental Developmental Index (of Bayley test)
m-l, medial-lateral direction
MU, movement units
PDI, Psychomotor Developmental Index (of Bayley test)
PT, preterm
GMs, general movements

Gradually it is getting clear that motor development in so-called "low-risk" preterm infants often differs from that in full-term infants. It is especially the development of postural control that is reported to be different (1). For instance, lowrisk preterm infants have been reported to show hyperextension of neck and trunk $(2,3)$, a reduced amount of rotation during crawling, a delayed onset of independent walking and a poor quality of early walking behavior $(4,5)$. At school age the dysfunctions in balance control are expressed as problems in standing on one leg (6), poor hopping and clumsy walking (7).

Received January 13, 2003; accepted June 27, 2003.

Correspondence: Bjørg Fallang, Oslo University College, Health Sciences, Physiotherapy Programme, Pilestredet 52, 0167 Oslo, Norway; e-mail bjorg.fallang@hf.hio.no

This study was supported by The Norwegian Fund for Postgraduate Training in Physiotherapy, Norway

DOI: 10.1203/01.PDR.0000088072.64794.F3
However, little is known on the etiology and developmental pathophysiology of these problems.

To acquire knowledge on the pathophysiology of postural behavior during skill acquisition at early age, specific methods have been applied. Two recent studies indicated that postural muscle activity of preterm children differs from that of full terms. One study (8) evaluated postural activity in response to platform perturbations in sitting children aged 1.5-4.5 years. They reported that preterm children with and without $\mathrm{CP}$, who had shown lesions of the periventricular white matter (PWM) on the neonatal ultrasound scans of the brain, exhibited a limited repertoire of postural adjustments. Preterm children with PWM lesions and those with normal ultrasound scans were less able to modulate postural muscle activity to initial sitting position. This modulatory capacity depends on the presence of internal representations, which are formed on the 
basis of prior experience (9). As the preterm children also showed an increased sensitivity to velocity dependent stretch, it was suggested that in preterm children, a form of control during which feedback processes dominate has replaced the normal balance between feedforward and feedbackward control of posture. The longitudinal study of Van der Fits et al. (10), in which postural muscle activity during reaching in various positions of full-term and preterm infants was assessed between the ages of 3 and $18 \mathrm{mo}$, confirmed the presence of a dysfunction in the capacity to modulate postural activity. The study also showed that preterm infants older than 4 mo activated more postural muscles during reaching than full term infants and that the postural adjustments of preterm infants were characterized by temporal disorganization. As yet it is unknown how the postural dysfunctions of preterm infants affect the quality of reaching behavior.

Recently we studied the relationship between postural control and reaching behavior in supine position in full-term infants (11). The study revealed that at 4 mo of age the total body Center of Pressure (COP) displacement occurs especially in the medial-lateral direction and that COP displacement decreases significantly with increasing age. The reaching movements were evaluated with kinematic analysis, such as the number of movement units (MU), which are determined as peaks in the velocity profile of the reaching hand (12), total reaching time and velocity. Like others $(13,14)$, we found that normal development is characterized by a decrease of the number of MU, a decrease in reaching time and an increase of the relative size of the first MU. The better reaching performance at 6 mo was related to a better postural stability. In another study we found that the kinematic quality of reaching movements of high-risk preterm infants without $\mathrm{CP}$ was less optimal than that of full-term infants (15). The aim of the present study is to assess whether this worse quality of reaching is related to a less adequate control of posture. Such knowledge is indispensable for the development of effective intervention strategies for preterms with problems in the control of reaching and prehension. Our specific aim was to evaluate postural behavior during reaching in high- and lowrisk preterm infants without cerebral palsy at the corrected ages of 4 and 6 mo. The ages of 4 and 6 mo were chosen because at 4 mo successful reaching is emerging and at 6 mo the first fast developmental phase of reaching has ended (13). The infants were investigated while they were lying on a forceplate. We addressed the following questions: 1) Do preterm infants at the corrected ages of 4 and 6 mo differ from full terms in postural activity during reaching in terms of displacement and $V_{\max }$ of COP? If they do 2) are any differences related to birth characteristics or morbidity during the stay in the neonatal ward, including mild abnormalities present on the ultrasound scan of the brain and the quality of general movements at the age of 4 mo? 3) Are any differences in COP behavior related to the infant's achievement on clinical test of neurodevelopmental performance, such as the scores on the Bayley scales (16), at the ages of 6 and $12 \mathrm{mo}$ ? 4) What are the relationships between the displacement and velocity of the body's COP and the kinematics of reaching in terms of number of $\mathrm{MU}$, hand displacement, velocity of hand displacement and kinematic reaching quality at 4 and 6 mo?

\section{METHODS}

Subjects. The preterm infants were recruited from three hospitals covering the city of Oslo (Ullevaal, Aker) and the nearest county (Akershus) at $36 \mathrm{wk}$ postmenstrual age. Gestational age at birth was based on the date of the last menstrual period and confirmed by ultrasound measurement during early pregnancy. The primary inclusion criteria were a birthweight below $1750 \mathrm{~g}$ and a mother speaking either a Scandinavian language or English. Infants with serious illness at $36 \mathrm{wk}$ or with cystic periventricular leukomalacia or intracranial hemorrhages extending into the parenchyma were excluded. This resulted in a group of 88 infants. After inclusion, two parents denied further participation, and families of four infants moved out of the area. Eight infants were excluded at a later stage because of serious illness or cerebral palsy, leaving the study with 74 infants. The absence of serious CNS conditions (cerebral palsy) in the infants included in the study was confirmed at a follow-up at one year consisting of a clinical examination and a Bayley assessment, and further during a recent follow-up at the age of five years, which included a detailed neurologic assessment according to Touwen (17). The group of 74 preterms was divided into a high-risk (HR) and a low-risk (LR) group. High-risk was assigned in case the infant had experienced one or more of the following risk factors: 1) Apgar score at $5 \mathrm{~min}<3,2)$ at least one of the following respiratory problems: a) respiratory illness needing surfactant-treatment, b) $>1 \mathrm{wk}$ of mechanical ventilation, c) pneumothorax, d) persistent ductus arteriosus. The diagnosis persistent patent ductus arteriosus was based on clinical and echocardiographic data (18). Four infants had a retinopathy of prematurity (ROP), one a grade 1, two a grade 2 and the fourth a grade 3 . None of the infants had a ROP plus disease (19).

We aimed at recording reaching movements of all infants at the corrected ages of 4 and 6 mo. The particular ages were chosen because at 4 mo the reaching is newly acquired. At the age of 4 mo successful kinetic recordings were obtained in 32 infants. No kinetic data were available in the rest due to absence of reaching behavior $(n=10)$, technical problems $(n$ $=13)$, artifacts in the forceplate data $(n=12)$, minor illnesses $(n=3)$ and family holidays $(n=4)$. At 6 mo successful recordings were obtained in 31 infants. Kinetic data were missing due to absence of reaching behavior $(n=1)$, technical problems $(n=19)$, artifacts in the forceplate data $(n=10)$, minor illnesses of the child $(n=6)$ or parental holidays $(n=$ 7). The neonatal characteristics of the 42 , respectively, 43 infants in whom no kinetic data could be recorded did not differ from those of the 32 , respectively, 31 preterms in whom the kinetics could be obtained at 4 and 6 mo. Neither did neonatal characteristics of the infants who could reach differ from the "nonreachers" at a particular age. The clinical data on the studied infants are summarized in Table 1.

Thirteen healthy infants born at term (FT), who participated in a similar project on the development of reaching and postural control, served as control infants (11). These infants who 
Table 1. Clinical characteristics of preterm infants assessed at 4 and 6 months

\begin{tabular}{|c|c|c|c|c|}
\hline \multirow[b]{2}{*}{ Infant characteristics } & \multicolumn{2}{|c|}{ High risk group } & \multicolumn{2}{|c|}{ Low risk group } \\
\hline & $\begin{array}{c}4 \text { months } \\
n=11\end{array}$ & $\begin{array}{c}6 \text { months } \\
n=10\end{array}$ & $\begin{array}{c}4 \text { months } \\
n=21\end{array}$ & $\begin{array}{c}6 \text { months } \\
n=21\end{array}$ \\
\hline Sex: M/F & $4 / 7$ & $6 / 4$ & $10 / 11$ & $10 / 11$ \\
\hline Birthweight in grams; median & 770 & 862 & 1250 & 1225 \\
\hline (Range) & $(560-1550)$ & $(650-1550)$ & $(890-1710)$ & $(890-1710)$ \\
\hline Gestational age at birth in weeks, median (range) & $27(24-34)$ & $28(24-34)$ & $30(26-34)$ & $31(26-32)$ \\
\hline SGA $n(\%)$ & $3(27)$ & $5(50)$ & $8(38)$ & $11(52)$ \\
\hline Mechanical ventilation $>1$ wk $(n)$ & 10 & 8 & 0 & 0 \\
\hline Ultrasound of brain available $(n)$ & 4 & 7 & 12 & 16 \\
\hline Normal US data $(n)$ & 3 & 5 & 8 & 10 \\
\hline IVH & 0 & 0 & 1 & 1 \\
\hline IVH + ventricles & 0 & 0 & 1 & 0 \\
\hline PVE & 1 & 1 & 0 & 1 \\
\hline Ventricles & 0 & 0 & 0 & 1 \\
\hline PVE + ventricles & 0 & 0 & 0 & 1 \\
\hline $\mathrm{PVE}+$ ventricles $+\mathrm{IVH}$ & 0 & 0 & 0 & 1 \\
\hline PVE + ventricles $+\mathrm{IVH}+$ small cysts & 0 & 1 & 2 & 0 \\
\hline Concurrent bodyweight in grams & 5320 & 8000 & 5600 & 6245 \\
\hline Median (range) & $(3500-6800)$ & $(6600-8300)$ & $(4600-8000)$ & $(4700-8750)$ \\
\hline Concurrent body length in $\mathrm{cm}$ & 58 & 67 & 60 & 64 \\
\hline Median (range) & $(48-66)$ & $(67-68)$ & $(42-65)$ & $(43-69)$ \\
\hline
\end{tabular}

SGA, small for gestational age; IVH, peri/intraventricular haemorrhage grade 1 or 2; PVE, periventricular echodensities lasting for at least 14 days; ventricles, ventriculomegaly.

were recruited at Ullevaal Hospital in Oslo, were born at a mean gestational age of $40 \mathrm{wk}$ and $6 \mathrm{~d}$ with a mean birthweight of $3878 \mathrm{~g}$ (SD \pm 442 ). The Ethics Committee of region 1 of Norway approved the study, and all parents signed informed consent before participation.

Procedure. The infants were placed in supine position on the forceplate on a folded towel, with the cranial-caudal orientation of the infant aligned parallel to the length of the forceplate, and with a toy at reaching distance above the chest. The kinetics was recorded by either an Amti force plate, or a Kistler force plate. Both plates were $40 \times 60 \mathrm{~cm}$ and measured ground reaction forces with a sampling frequency of $1000 \mathrm{~Hz}$. The kinematics of the reaching movements was recorded with either the MacReflex movement analysis system (Qualisys, Sweden) or the Peak Performance Movement Analysis System (Peak Performance Technologies, Inglewood, CO, U.S.A.). Four cameras were mounted perpendicular to each other, allowing for a view of both right and left side (3-D) for the collection of kinematic data. To record the reaching movements spherical reflective markers with a diameter $2.5 \mathrm{~cm}$ were attached to the back of both hands and the toy. Three successful reaching movements (contact with the toy) were recorded per infant. Care was taken that the child was in a noncrying, alert behavioral state during reaching. The force plate and the kinematic data were recorded simultaneously and time-coupled for $15 \mathrm{~s}$ (11). Table 2 shows the number of trials analyzed.

Neonatal morbidity of the children was collected in a standardized way. In $67 \%$ of the infants' data on serial neonatal ultrasound scans of the brain were available. The scans were made with a $7.5 \mathrm{MHz}$ transducer. Periventricular hemorrhages and echo densities were classified according to the methods of Levene (20) and Jongmans (21) (Table 1).

To assess the quality of brain function at early age, the quality of general movements (GMs) in supine position was assessed at 4 mo of age. By analyzing video-recordings of spontaneous behavior (3-5 $\mathrm{min}$ ), the quality of GMs was classified into normal, mildly abnormal or definitively abnormal movements according to Hadders-Algra et al. (22) by the last author who at the time of GM-assessment was blind to the infants' clinical status, reaching and postural control data. Inter-rater reliability of this assessment is high (kappa 0.81) (22) The infant's level of motor (PDI) and mental development (MDI) was quantified with the Bayley Scales of Infant Development (16) at the corrected ages of 6 and $12 \mathrm{mo}$. The assessors were blind to neonatal histories and high- and lowrisk allocation.

Analysis. The data from the two forceplate and the two kinematic systems were processed in an identical way. They

Table 2. Summary of number of subjects and reaching trials included in the study

\begin{tabular}{|c|c|c|c|c|c|c|}
\hline \multirow[b]{2}{*}{ Group } & \multicolumn{2}{|c|}{ Adequate COP data at 4 and 6 months } & \multicolumn{2}{|c|}{ Adequate COP data at 4 months only } & \multicolumn{2}{|c|}{ Adequate COP data at 6 months only } \\
\hline & Subjects $(n)$ & Total number of trials analyzed & Subjects $(n)$ & Total number of trials analyzed & Subjects $(n)$ & Total number of trials analyzed \\
\hline PT-LR & 10 & 17 and 23 & 11 & 24 & 11 & 23 \\
\hline PT-HR & 3 & 5 and 4 & 8 & 15 & 7 & 17 \\
\hline FT & 7 & 22 and 22 & 1 & 1 & 3 & 10 \\
\hline
\end{tabular}

COP, center of pressure; PT-LR, low-risk preterm; PT-HR, high-risk preterm; FT, fullterm.

At 4 mo 32 PT infants were tested, and 31 infants at 6 mo, note that some infants were only tested at 4 mo or only at 6 mo of age. 
were exported for further analysis to a custom made program (M. Guttormsen, Norwegian University of Sports and Physical Education, Oslo). The COP data were filtered with a 2nd order Butterworth filter at $20 \mathrm{~Hz}$. COP was calculated separately in two directions: cranial-caudal (c-c) and medial-lateral (m-l). The following parameters were calculated: 1 ) The total COP displacement during the reaching movement, defined as the real distance traveled by COP during the reaching period. 2) The $V_{\max }$ of COP displacement, determined from the derivative of the displacement curve. 3) The number of directional changes (oscillations) in the COP displacement in c-c and m-1 direction. The oscillations, which consisted of two directional changes in the COP displacement of $>2 \mathrm{~mm}$, were visually identified on the graphical displacement curve. 4) A directional index of the three COP-parameters was calculated by dividing the COP value in $\mathrm{m}-\mathrm{l}$ direction by the one in c-c direction.

Reaching onset was defined as the video frame when the reaching arm started to move toward the toy and reaching ended when the hand touched the toy, which was indicated by an increase in the velocity profile of the toy. The MUs were distinguished with the help of the hand velocity peak profile (13). The MUs were defined from the velocity profile by three characteristics: 1 ) at the beginning of the unit the velocity had to increase by $20 \mathrm{~mm} / \mathrm{s} ; 2$ ) the velocity peak of the MU had to be higher than $10 \%$ of the $V_{\max }$ for the whole trial; 3) the local maximum of a velocity peak was defined as the highest peak between three frames to each side. The kinematic data were filtered with a zero phased forward/backward 2nd order Butterworth filter with cut-off frequency of $6 \mathrm{~Hz}$. The analysis of the reaching movements focused on 1 ) the number of movement units (MU). 2) The length of the first MU relative to total movement path; 3) the duration of the first MU relative to total movement time; 4) maximal hand velocity during each reach; $5)$ total movement time; 6 ) the quality of reaching where reaching movements were classified on the basis of $V_{\max }$ and the number of MU. Based on the performance of the full-term infants (15), the reaching movements at 4 mo were considered to be optimal, when they had a medium velocity (301-800 $\mathrm{m} / \mathrm{s}$ ), irrespective the number of MU. At 6 mo the criteria for optimal reaching movements were a medium velocity combined with the presence of only a few, i.e. less than 3.5 MU (15).

Statistics. For the various COP and kinematic parameters individual median values were calculated for each infant at each age. To evaluate the effect of age on postural development, and the differences in COP behavior between the three groups, the nonparametric Mann-Whitney test was used. Mann-Whitney was used instead of Wilcoxon because of missing data at both ages. Wilcoxon was performed for comparisons between COP displacement directions.

For the analysis of relationships between neonatal condition and COP-behavior, the neonatal data were dichotomized (condition present or absent) and analyzed with the Mann-Whitney test. The relationships between COP-behavior and a) the reaching behavior and b) performance on the clinical tests were evaluated with Spearman rank correlation. The relative importance of anthropometrics and group (FT or PT) membership on COP behavior was calculated by multivariate regression analyses. Statistical calculations were performed with SPSS version 11. Differences with $p$-values $<0.05$ were considered to be statistically significant (two tailed). For the large number of correlations the level of significance was set at 0.01 to avoid the risk of making a type I error.

\section{RESULTS}

Total body center of pressure behavior in preterm infants. COP behavior of the preterm infants differed markedly from that of the full terms, while that of LR and HR preterms did not differ significantly from each other. At 4 mo the total displacement of COP and the number of COP oscillations in both c-c and $\mathrm{m}-1$ directions of the preterm infants were significantly less than those of the full term infants (Fig. 1 and 2). Also maximum COP velocity differed between the groups, with the full cranial-caudal direction

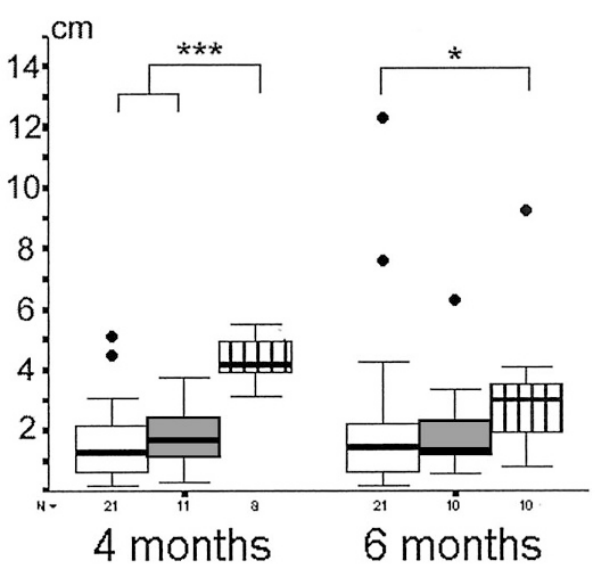

medial-lateral direction

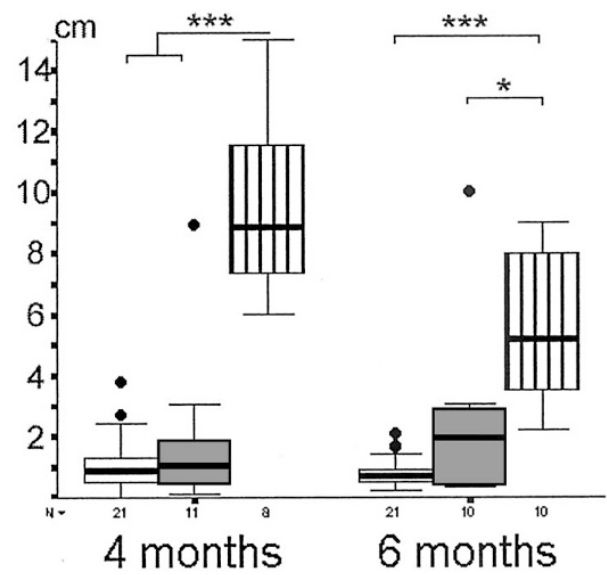

Figure 1. Total COP displacement in the cranial-caudal and medial-lateral direction at 4 and 6 mo in low-risk preterm (LR) open boxes, high-risk preterm (HR) $=$ gray boxes, and full term (FT) infants = striped boxes. The horizontal bars indicate the group median values, the boxes the interquartile values, the vertical lines denote the ranges and the outliers are indicated by dots. Mann-Whitney: ${ }^{*} p<0.05,{ }^{*} p<0.01,{ }^{* * *} p<0.001$. 
cranial-caudal direction

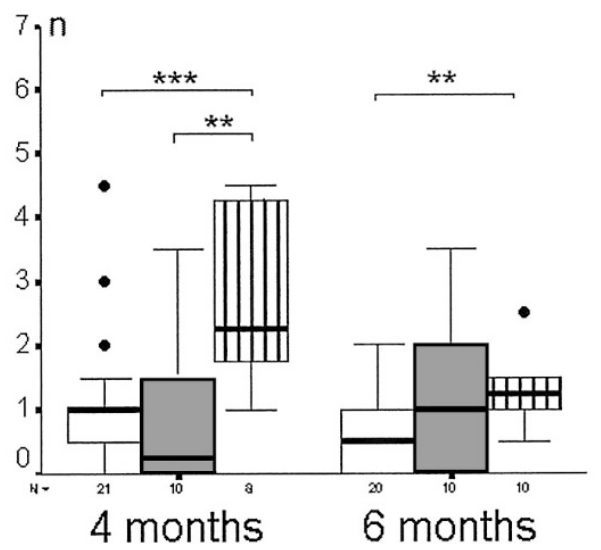

medial-lateral direction

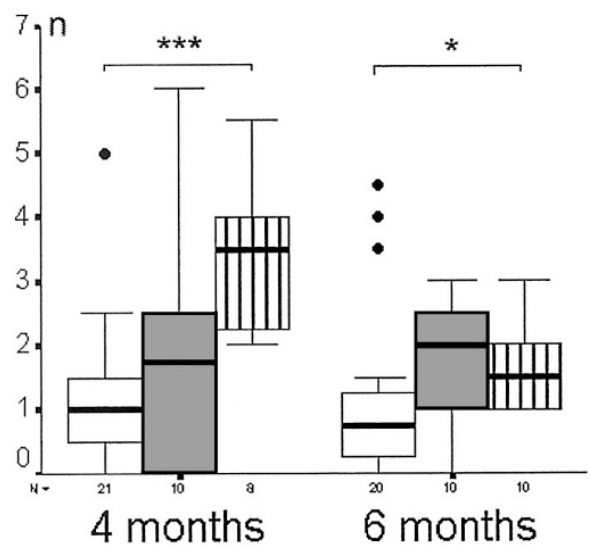

Figure 2. Number of COP oscillations in the cranial-caudal and medial-lateral direction at 4 and 6 mo in low-risk preterm $(\mathrm{LR})=$ open boxes, high-risk preterm $(\mathrm{HR})=$ gray boxes, and full term (FT) infants = striped boxes. The horizontal bars indicate the group median values, the boxes the interquartile values, the vertical lines denote the ranges and the outliers are indicated by dots. Mann-Whitney: ${ }^{*} p<0.05, * * p<0.01, * * * p<0.001$.

terms exhibiting higher COP velocities in both directions than the preterms (Fig. 3).

In the full term infants COP behavior changed significantly between 4 and 6 mo. (Fig. 1 and 2). Median displacement changed from 4.2 to $3.0 \mathrm{~cm}$ in the c-c direction $(p=0.02)$ and from 8.9 to $5.2 \mathrm{~cm}$ in the $\mathrm{m}-1$ direction $(p=0.03)$. Likewise the median number of COP oscillations decreased with increasing age from 2.25 to 1.25 in the $\mathrm{c}-\mathrm{c}$ direction $(p=0.01)$ and from 3.5 to 1.5 in the m-1 direction $(p=0.001)$, while $V_{\max }$ of COP did not change with age (Fig. 3). In both preterm groups COP behavior did not change significantly between 4 and 6 mo.

Despite the fact that the full term infants did show an age-related reduction of COP motility and the preterm infants did not, preterm infants continued to have lower values in total COP displacement (Fig. 1) and in $V_{\max }$ (Fig. 3) than full term infants at $6 \mathrm{mo}$, (c-c direction: FT: $8.5 \mathrm{~cm} / \mathrm{s}$, LR: $4.0 \mathrm{~cm} / \mathrm{s}, \mathrm{HR}$ :
$3.6 \mathrm{~cm} / \mathrm{s}$; m-1 direction: FT: $16.5 \mathrm{~cm} / \mathrm{s}$, LR: $2 \mathrm{~cm} / \mathrm{s}, \mathrm{HR}: 4.7$ $\mathrm{cm} / \mathrm{s}$; FT versus LR $p=0.001(\mathrm{~m}-1)$ and $p=0.02$ (c-c) and FT versus $\mathrm{HR}, p=0.004$ (m-l) and $p=0.04$ (c-c)). LR infants showed significantly less oscillations than full term infants at 6 mo, $(p=0.03$ in $\mathrm{m}-1$ and $p=0.007$ in c-c) but the HR infants did not differ from the full term infants (Fig. 2).

Another difference in COP behavior between full term and preterm infants was observed in the relation between displacement in medial-lateral and cranial-caudal direction. At 4 mo the full term infants showed longer COP displacements and higher COP velocities in the $\mathrm{m}-\mathrm{l}$ than in the $\mathrm{c}-\mathrm{c}$ direction (displacement and velocity $p=0.01$, Fig. 1 and 3 ). In contrast, the LR infants at 4 mo had shorter displacements and lower velocities in the $\mathrm{m}-1$ than in the c-c direction $(p=0.003$ and $p$ $=0.05$, respectively). The HR infants did not show directional differences in COP-behavior at 4 mo. At 6 mo, this group cranial-caudal directions

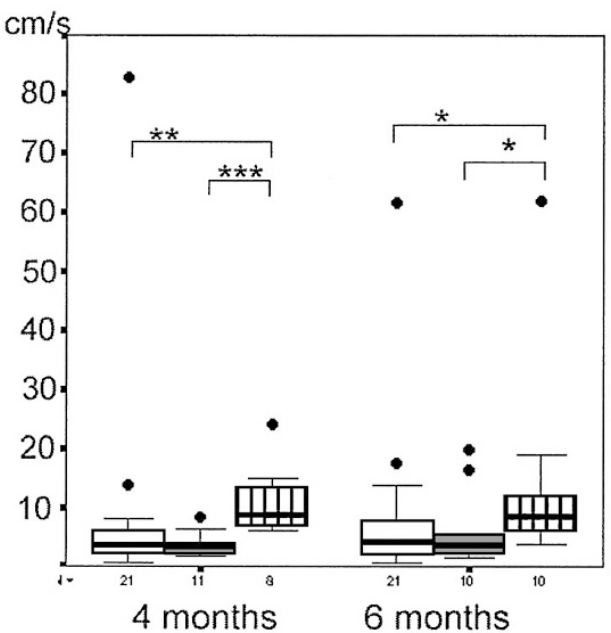

medial-lateral directions

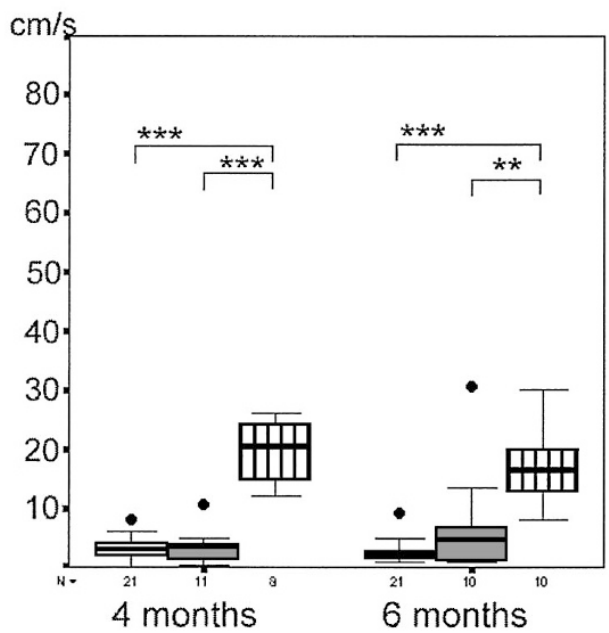

Figure 3. $V_{\max }$ in the cranial-caudal and medial-lateral direction at 4 and 6 mo in low-risk preterm (LR) $=$ open boxes, high-risk preterm (HR) $=$ gray boxes, and full term (FT) infants = striped boxes. The horizontal bars indicate the group median values, the boxes the interquartile values, the vertical lines denote the ranges and the outliers are indicated by dots. Mann-Whitney: ${ }^{*} p<0.05,{ }^{*} p<0.01,{ }^{* * *} p<0.001$. 
specific directional behavior of COP was virtually the same. The full-terms continued to have longer displacements $(p=$ $0.009)$ and somewhat higher velocities $(p=0.07)$ in the m-1 than in the c-c direction. In the LR group the opposite behavior persisted: shorter displacements $(p=0.005)$ and lower velocities $(p=0.002)$ in the $\mathrm{m}-\mathrm{l}$ direction. The HR infants continued to perform without directional differences.

Clinical data and COP behavior in the preterm infants. $\mathrm{We}$ wondered whether the differences in COP behavior between the full term and preterm infants could be explained by differences in anthropometrics. At 4 mo the full term infants were significantly heavier $(p=0.001)$ and longer $(p=0.001)$ than the preterm infants (median weights $\mathrm{FT}=7350 \mathrm{~g}$, LR $=$ $5600 \mathrm{~g} \mathrm{HR}=5320 \mathrm{~g}$; median lengths $\mathrm{FT}=65.5 \mathrm{~cm}, \mathrm{LR}=60$ $\mathrm{cm}, \mathrm{HR}=58 \mathrm{~cm})$. At $6 \mathrm{mo}$ the full term infants were still heavier $(p=0.001)$ and longer $(p=0.02)$ than the LR infants, but the difference in body size between the full term and HR infants had disappeared (median weights FT $=8235 \mathrm{~cm}, \mathrm{LR}=$ $6245 \mathrm{~g}, \mathrm{HR}=8000 \mathrm{~g}$; median lengths $\mathrm{FT}=68 \mathrm{~cm}, \mathrm{LR}=64.5$ $\mathrm{cm}, \mathrm{HR}=67.5 \mathrm{~cm})$. Regression analyses revealed that groupmembership (full term or preterm) outweighed the effect of body size, leaving no significant contribution of body weight and body length on COP-behavior.

Birthweight, being small-for-gestational age, i.e. having a birthweight at or below the tenth percentile, or the need of mechanical ventilation were not related to COP behavior. A gestational age of less than $28 \mathrm{wk}$ at birth did not affect COP behavior at $4 \mathrm{mo}$, but it did affect COP behavior at $6 \mathrm{mo}$. The infants born before 28 wk showed a longer COP displacement $(p=0.003)$ and a higher velocity $(p=0.01)$ in the $\mathrm{m}-1$ direction and more oscillations in c-c direction $(p=0.04)$ than the infants born at or after $28 \mathrm{wk}$. The few preterm infants who had shown mild abnormalities on the neonatal ultrasound scans (Table 1) did not differ significantly in their COP behavior from those who had had a normal ultrasound scan.

The quality of GMs in the preterm infants at 4 mo was not related to postural behavior at $4 \mathrm{mo}$, but it was related to postural behavior at $6 \mathrm{mo}$. None of the infants had definitely abnormal GM's at 4 mo. Preterm infants with normal GMs ( $n$ $=20$ ) showed a smaller COP displacement in the m-l direction $(p=0.04)$ and less oscillations in the c-c direction $(p=0.009)$ than infants with mildly abnormal $(n=11)$ GMs. In addition, the infants with normal GMs had lower values of the directional indices of displacement and velocity, i.e. lesser degrees of COP-behavior in the m-l direction, than infants with mildly abnormal GMs $(p=0.02)$.

COP behavior at 4 and 6 mo was not related to the Bayley scores at 6 or 12 mo in any of the three groups. In summary, our data indicate that COP behavior at 4 month was not related to clinical characteristics, but COP behavior at 6 mo was. Less COP motility at 6 mo was related to a gestational age at birth of $28 \mathrm{wk}$ or more and to the presence of normal GMs at $4 \mathrm{mo}$.

COP behavior and reaching variables. In the 4 mo old full term infants COP behavior and reaching characteristics were related. This was indicated by a significant relationship between the COP displacement in the m-l direction and the duration of reaching, between the number of oscillations in $\mathrm{m}-1$ direction and the duration of reaching and the number of MU. The number of oscillations in c-c direction was related to the length and time of 1st MU (Table 3).

Despite the rather small displacements seen in the preterm infants, COP displacement was related to reaching activity. In the 4 mo old LR preterm infants COP displacement in m-1 direction was correlated to the relative length and duration of the first MU. In the HR group COP displacement in $\mathrm{m}-1$ direction was related to the duration of reaching and the number of MU. When the two preterm groups were pooled, similar relationships were found between COP behavior and the kinematics of reaching (Table 3). None of the COPparameters at 4 mo were related to the quality of reaching movements at that age.

At 6 mo the quantitative reaching variables and $\mathrm{COP}$ were less coupled than at $4 \mathrm{mo}$. The only relationship that was found was the one between reaching velocity and COP velocity in m-1 direction. This relationship was statistically significant in the LR infants, approached significance in the full term infants and was not significant in the HR infants (Table 3). In contrast to the virtual absence of a relationship between COP behavior and the quantitative kinematic parameters of reaching, COP behavior at 6 mo was related to the quality of reaching. The LR infants who showed an optimal kinematic reaching quality had a shorter COP displacement in both directions $(p=0.02)$, lower COP velocities (c-c: $p=0.02$ and $\mathrm{m}-1: p=0.04)$, and less oscillations (m-l: $p=0.001)$. In the HR and full term

Table 3. Correlations between COP behaviour and kinematics of reaching

\begin{tabular}{|c|c|c|c|c|c|c|c|c|}
\hline \multirow[b]{2}{*}{$\mathrm{COP}^{*}$ reaching } & \multicolumn{4}{|c|}{4 months } & \multicolumn{4}{|c|}{6 months } \\
\hline & FT & LR & HR & All PT & $\mathrm{FT}$ & LR & HR & All PT \\
\hline disp $\mathrm{m}-1 *$ duration & $0.86^{*}$ & - & $0.77 *$ & $0.48^{*}$ & - & - & - & - \\
\hline displ $\mathrm{m}-1 *$ rel length $1^{\text {st }} \mathrm{MU}$ & - & $-0.62 *$ & & $-0.46^{*}$ & - & - & - & - \\
\hline displ $\mathrm{m}-1 *$ rel duration $1^{\text {st }} \mathrm{MU}$ & - & $-0.58 *$ & - & $-0.54^{*}$ & - & - & - & - \\
\hline vel $\mathrm{m}-1 *$ duration & - & - & - & - & - & - & - & - \\
\hline osc $\mathrm{m}-1 *$ duration & $0.85^{*}$ & - & - & - & - & - & - & - \\
\hline osc $\mathrm{m}-1 *$ number of $\mathrm{MU}$ & $0.88^{*}$ & - & - & - & - & - & - & - \\
\hline osc c-c * rel length $1^{\text {st }} \mathrm{MU}$ & $-0.87^{*}$ & - & - & - & - & - & - & - \\
\hline osc c-c $*$ rel duration $1^{\text {st }} \mathrm{MU}$ & $-0.82 *$ & - & - & - & - & - & - & - \\
\hline
\end{tabular}

Spearman rank correlation, $* p<0.01$.

COP, Disp = displacement; osc, number of oscillations; vel, maximum velocity. Reaching: MU, movement units; max vel, maximum velocity, rel, relative. 
groups COP behavior and the quality of reaching were not related. This was due to the fact that the majority in the HR group showed nonoptimal reaching movements and the majority in the full term group showed reaching movements with an optimal kinematic quality. When both preterm groups were pooled, the infants with optimal reaching quality had a shorter displacement in both directions $(p=0.02$ and 0.03 , respectively) and a lower velocity and less oscillations in the m-1 direction ( $p=0.02$ and $p=0.001$, respectively).

\section{DISCUSSION}

The present study demonstrated that postural behavior of preterm infants during early infancy differs markedly from that of full term infants. In contrast to the mobile COP behavior exhibited by full term infants during goal directed reaching in supine, preterm infants present with a "still" or nearly motionless postural behavior. The relatively fixed postural behavior of the preterms could not be explained by the fact that their body proportions were smaller than that of the full-terms.

The major question is whether this "still" COP behavior can be regarded as an expression of neurologic dysfunction or whether it should be interpreted as a profitable form of adaptation to the extrauterine environment by the very preterm infant. Our data, in particular the COP-data at 6 mo, favor the second explanation. First, "still" COP behavior at 6 mo was related to the presence of normal GMs at 4 mo. Previous studies indicated that the quality of GMs reflects the integrity of brain function $(23,22)$. Thus, "still" COP-behavior at 6 mo seems to be related to relatively normal brain function. Second, less postural motility during reaching at 4 mo in supine was related to better quantitative characteristics of reaching, such as movements with a shorter duration, fewer MUs and a proportionally larger transport unit, and "still" COP behavior at 6 mo was related to a better quality of reaching. Third, a gestational age at birth of less than $28 \mathrm{wk}$, which in general is related to a less favorable neurologic outcome than birth after that age (24, 25 ), was related to more mobile COP activity at six months.

Thus we may argue that the "still" COP behavior, especially at $6 \mathrm{mo}$ of age is a form of neuromotor adaptation in response to the early demands of the extrauterine environment. An adaptation which consists of an active reduction of the degrees of freedom in the body, in particular in the medial-lateral direction, and which is associated with an excess of activity in the postural muscles (10). An adaptation which can be interpreted as functionally adequate in early infancy, as it results in better reaching possibly by securing the position of the head and shoulders as a stable frame of reference. But it is conceivable that this early postural adaptation may be related to less adequate motor behavior during further development, i.e. to the reduced capacity of preterm children to fine tune postural behavior to specific conditions and to learn from motor experience as found by others $(10,8)$. This might mean that the postural behavior of the preterm infant, even though it is favorable at early age, is a marker of later neuromotor dysfunction. In this respect it is interesting that COP-behavior was not related to mild abnormalities present on the neonatal ultrasound scans of the brain. Of course it should be kept in mind that ultrasound data were only available in about $60 \%$ of the infants. Nevertheless, a similar absence of relationships between ultrasound findings and minor developmental disorders has been reported before $(8,10)$. It could be surmised that the nonoptimal motor behavior of some preterm infants is an expression of mild abnormalities of the brain, which escape the ultrasonographer's eye. Evidence is accumulating that the minor developmental abnormalities could be related to stress perceived in the extrauterine environment before term age (26, 27). Significant increases in the catecholamines indicated that relatively invasive nursing procedures, such as endotracheal suction and chest physiotherapy, but also noninvasive handling procedures such the changing of a nappy, are stressful for the preterm infant $(28,29)$. Animal research has indicated that exposure to stress during early development can induce permanent alterations in the structure and function of the CNS. Studies in rats showed that prenatal stress resulted in changes in serotonergic and noradrenergic activity in the cerebral cortex and alterations in dopaminergic activity in the striatum and prefrontal cortex (30). Other studies indicated that alterations in the development of the monoaminergic systems can induce an impaired development of the maps of body representation in the primary somatosensory cortex (31). In this respect it is interesting to note that a recent study of Hellerud and Storm (32) indicated that preterm infants differ significantly from term infants in response to tactile handling, both at term age and at 3 mo post-term.

In conclusion, our study indicates that preterm infants without cerebral palsy exhibit a "still" postural behavior during reaching in supine, which is not present in full-term infants. The "still" postural behavior is functionally adequate at early age, as it is associated with good reaching performance. Yet, we hypothesize that the "still" postural behavior is a motor adaptation, which is only temporarily and in the supine position adequate. It may be the result of stress-induced alterations in the central processing of somatosensory information and therefore be followed by less appropriate motor learning and development at later age.

Acknowledgments. We thank physical therapists and neonatologists at Aker, Ullevaal, Akershus University hospitals for recruiting the infants and providing clinical information. Vidar Jacobsen and his team at the Norwegian University of Sports and Physical Education, Oslo, is thanked for skillful technical assistance in the collection of kinematic and kinetic data, Kristin Gillebo for participation in the Bayley assessments.

\section{REFERENCES}

1. Ornstein M, Ohlsson A, Edmonds J, Asztalos E 1991 Neonatal follow-up of very low birthweight/extremely low birthweight infants to school age: a critical overview Acta Paediatr Scand 80(8-9):741-748

2. Touwen BC, Hadders-Algra M 1983 Hyperextension of neck and trunk and shoulder retraction in infancy - a prognostic study Neuropediatrics 14:202-205

3. de Groot L, Hopkins B, Touwen BC, Vanderhoek AM 1993 Development of muscle power in preterm infants - individual trajectories after term age. Neuropediatrics 24:68-73

4. de Groot L, Hopkins B, Touwen BC 1997 Motor asymmetries in preterm infants at 18 weeks corrected age and outcome at 1 year. Early Hum Dev 48:35-46

5. Gorga D, Stern FM, Ross G, Nagler W 1988 Neuromotor development of preterm and full-term infants. Early Hum Dev 18:137-149 
6. Forslund M, Bjerre I 1989 Follow-up of preterm children I. Neurological assessment at 4 years of age. Early Hum Dev 20: 45-66

7. Sommerfelt K, Ellertsen B, Markestad T, 1993 Personality and behaviour in eightyear-old, non-handicapped children with birth weight under 1500g. Acta Pædiatr 82: $723-728$

8. Hadders-Algra M, Brogren E, Katz-Salamon M, Forssberg H 1999 Periventricular leucomalacia and preterm birth have different detrimental effects on postural adjustments. Brain 122:727-740

9. Hadders-Algra M, Brogren E, Forssberg H 1996 Training affects the development of postural adjustments in sitting infants. J Physiol 493:289-298

10. Van der Fits I, Hadders-Algra M 1999 Development of postural adjustments during reaching in preterm infants. Pediatr Res 46:1-7

11. Fallang B, Saugstad OD, Hadders-Algra M 2000 Goal directed reaching and postural control in supine position in healthy infants. Behav Brain Res 115:9-18

12. von Hofsten C 1979 Development of visually directed reaching: The approach phase. J Hum Mov Stud 5:160-168

13. von Hofsten C 1991 Structuring of early reaching movements: A longitudinally study. J Mot Behav 23:280-292

14. Thelen E, Corbetta D, Kamm K, Spencer JP, Schneider K, Zericke RF 1993 The transition to reaching: mapping intention to intrinsic dynamics. Child Dev 64:1058 1098

15. Fallang B, Saugstad OD, Grøgaard J, Hadders-Algra M 2003 Kinematic quality of reaching movements in preterm infants. Pediatr Res 53:1-7

16. Bayley N 1993 Bayley Scales of Infant Development, 2nd Ed. Psychological Corporation Hartcourt Brace \& Company, San Antonio, TX, pp 1-367

17. Touwen BC 1979 Examination of the Child with Minor Neurological Dysfunction. 2nd Ed. Clin Dev Med 71, Spastics International Medical Publications, William Heinemann Medical Books, London, pp 1-123

18. Evans N 1993 Diagnosis of patent ductus arteriosus in the preterm newborn. Arch Dis Child 68:58-61

19. Committee for the classification of retinopathy of prematurity: International classification of retinopathy of prematurity 1984 Arch Ophthalmol 102:1130-1134
20. Levene MI, Fawer C-L, Lamont RF 1982 Risk factors in the development of intraventricular haemorrhage in the preterm neonate. Arch Dis Child 57:410-417

21. Jongmans M, Henderson S, De Vries L, Dubowitz L 1993 Duration of periventricular densities in preterm infants and neurological outcome at six years of age. Arch Dis Child 69:9-13

22. Hadders-Algra M, Mavinkurve-Groothuis AMC, Groen SE, Stremmelaar EF, Martijn A, Butcher PR 2003 Quality of general movements and the development of minor neurological dysfunction at toddler and school age. Clin Rehabil, in press

23. Prechtl HFR, Cioni G, Einspieler C, Bos AF, Ferrari F 2001 Role of vision on early motor development: lessons from the blind. Dev Med Child Neurol 43:198-201

24. Hagberg B, Hagberg G, Beckung E, Uvebrant P 2001 Changing panorama of cerebra palsy in Sweden. VIII. Prevalence and origin in the birth year period 1991-94. Acta Paediatr 90:271-277

25. Drummond PM, Colver AF 2002 Analysis by gestational age of cerebral palsy in singleton birth in north-east England 1970-94. Paediatr Perinat Epidemiol 16:172-180

26. Hadders-Algra M 2003 Developmental coordination disorder: is clumsy motor behaviour caused by a lesion of the brain at early age? Neural Plast, in press

27. Grunau R, 2002 Early pain in preterm infants. A model of long-term effects. Clin Perinatol 29:373-394

28. Greisen G, Frederiksen PS, Hertel J, Christensen NJ 1985 Catecholamine response to chest physiotherapy and endotracheal suctioning in preterm infants. Acta Pediatr Scan 74:525-529

29. Lagercrantz H, Nilsson E, Redham I, Hjemdahl P 1986 Plasma-catecholamines following nursing procedures in a neonatal ward. Early Hum Dev 14:61-65

30. Weinstock M 2001 Alterations induced by gestational stress in brain morphology and behaviour of the offspring. Prog Neurobiol 65:427-451

31. Cases O, Lebrand C, Giros B, Vitalis T, De Maeyer E, Caron MG, Price DJ, Gaspar P, Seif I 1998 Plasma membrane transporters of serotonin, dopamine, and norepinephrine mediate serotonin accumulation in atypical locations in the developing brain of monoamine oxidase A knock-outs. J Neurosci 18:6914-6927

32. Hellerud BC, Storm H 2002 Skin conductance and behaviour during sensory stimulation of preterm and term infants. Early Hum Dev 70:35-46 